\title{
GROUPS WITH SMALL CONJUGACY CLASSES
}

\author{
G. A. HOW AND F. C. CHUANG
}

\begin{abstract}
A group satisfies property (*) iff every conjugacy class has size not greater than 2. This paper proves properties of this type of group and conclude that it is a central product of an abelian group with 2 -groups that are "almost" extra special.
\end{abstract}

\section{Introduction} field.

All groups considered are finite groups. All characters are over the complex number

Let us say that a group satisfies property. (*) iff every conjugacy class of $G$ has size not greater than 2. This is obviously equivalent to the condition that $\forall x \in G$, $\left[G: C_{G}(x)\right] \leq 2$. This paper is to characterize all groups with property $(*)$.

In Section II, the problem of characterizing all groups with property $(*)$ is reduced to characterizing all such 2-groups, and some examples are given.

In Section III, we show the strong similarity between 2-groups satisfying property $(*)$ and the extra special 2-groups. Our conclusion is:

A group satisfying property $(*)$ is the direct product of an abelian group with an "almost" extra special 2-group.

II.

We reduce the problem of characterizing all groups with property $(*)$ to characterizing all such 2-groups. Two lemmas are needed.

Lemma 1. If $G$ satisfies property (*), then

(1) All subgroups of $G$ also satisfy property $(*)$

(2) All Sylow subgroups of odd order of $G$ are abelian

Proof. Let $H \leq G, \forall x \in H,\left[G: C_{G}(x)\right] \leq 2$ as $G$ satisfies property (*). If $H \subseteq C_{G}(x)$, then $\left[H: C_{H}(x)\right.$ is clearly 1 . If $H \nsubseteq C_{G}\left(x\right.$ then $H C_{G}(x)=G$ and clearly in this case $\left[H: C_{H}(x)\right] \leq\left[G: C_{G}(x)\right] \leq 2$. This proves $(1)$.

Received July 20, 1998; revised December 30, 1998.

1991 Mathematics Subject Classification. 20D99.

Key words and phrases. Conjugacy classes, extra special p-groups. 
Sylow subgroup $P$ of $G$ satisfies property $(*)$ by (1). Thus $\forall x \in P,\left[P: C_{P}(x)\right] \leq 2$. If $P$ has odd order, the only possibility is $\left[P: C_{P}(x)\right]=1$.This proves $(2)$

Lemma 2. If $G$ satisfies property $(*)$, then $G$ is nilpotent of class two. class 2 .

Proof. $\forall x \in G,\left[G: C_{G}(x)\right] \leq 2$ implies that $G^{\prime} \subseteq Z(G)$. Thus $G$ is nilpotent of

Lemma 2 asserts that $G$ is a direct product of all its Sylow subgroups. By Lemma 1 , all Sylow subgroups of odd orders are abelian. So we conclude that $G$ is a direct product of an abelian group with a 2 -group satisfying property $(*)$. Thus, this reduces the problem to characterizing 2 -group with property $(*)$.

Theorem 1. Let $G$ be a non-abelian 2-group. G satisfies property (*) iff $\left|G^{\prime}\right|=2$.

Proof. Assume one conjugacy class of $G$ has size greater than 2 . Let $x, y, z$ be its distinct elements. Then there exists $g_{1}, g_{2} \in G$ such that $y=g_{1}^{-1} x g_{1}, z=g_{2}^{-1} x g_{2}$. These give rise to two distinct nonidentity elements of $G^{\prime}$, namely, $y x^{-1}=g_{1}^{-1} x g_{1} x^{-1}$ and $z x^{-1}=g_{2}^{-1} x g_{2} x^{-1}$. We have prove that $\left|G^{\prime}\right|=2$ implies $G$ satisfies property $(*)$.

Conversely, assume $G$ satisfies property (*). Let $|G|=2^{n},\left|G^{\prime}\right|=2^{\alpha},|Z(G)|=2^{\beta}$. Each element in $Z(G)$ forms a conjugacy class, and every two elements outside $Z(G)$ form a conjugacy class. Thus $G$ has $\frac{|Z(G)+| G \mid}{2}$ conjugacy class, and $\frac{|Z(G)+| G \mid}{2}$ irreducible complex characters. Using the equality for character degree $\sum_{\chi \in \operatorname{Irr}(G)} \chi(1)^{2}=|G|$, and the fact that $G$ has $\left[G: G^{\prime}\right]$ linear characters, we get

$$
\begin{array}{ll} 
& {\left[G: G^{\prime}\right]+\left(\frac{|G|+|Z(G)|}{2}-\left[G: G^{\prime}\right]\right) 4 \leq|G|} \\
\text { i.e. } \quad 2^{n-\alpha}+\left[\frac{2^{n}+2^{\beta}}{2}-2^{n-\alpha}\right] 4 \leq 2^{n}
\end{array}
$$

This forces $\alpha=1$, and so $\left|G^{\prime}\right|=2$.

By Theorem 1, all extra special 2-groups satisfy property $(*)$, but the converse is not true, since any abelian group obviously satisfies property $(*)$. Even if we assume $G$ to be directly indecomposable, the converse is still not true. In fact, take $A=\langle x\rangle$, the cyclic group of order $2^{n}$, and extend it by a 2-cycle $\sigma$ where $x^{\sigma}=x^{2^{n-1}+1}$. The group obtained has order $2^{n+1}$ with commutator subgoup of size 2 . Thus it has property (*), yet it is directly indecomposable and not extra special.

III.

Some basic character theory are needed here,

Theorem A. (Isaac) [3] Theorem 6.18

Let $G$ be a group, $K / L$ be an elemetary abelian chief factor of $G$. Let $\chi \in \operatorname{Irr}(K)$ and suppose that $\chi$ is $G$-invariant. Then one of the following holds:

(1) $\chi_{L} \in \operatorname{Irr}(L)$ 
(2) $\chi_{L}=e \theta$ where $\theta \in \operatorname{Irr}(L)$ and $e^{2}=[K: L]$

(3) $\chi_{L}=\sum_{i=1}^{t} \theta_{i}$ where $t=[K: L]$ and the $\theta_{i} \in \operatorname{Irr}(L)$ are distinct.

Theroem. B. (Itô) [1] Theorem 6.15

Let $A$ be any abelian normal subgroup of $G$. Then $\forall \chi \in \operatorname{Irr}(G), \chi(1) \mid[G: A]$.

Theorem C. [3] Theorem 6.11

Let $H \leq G$ and $\theta$ be an irreducible character of $H$ which is invariant in $G$. Let $I_{G}(\theta)$ be the inertial subgroup of $\theta$. Then $\exists \zeta \in \operatorname{Irr}\left(I_{G}(\theta)\right.$ such that $\zeta^{G} \in \operatorname{Irr}(G)$ and $\zeta_{1_{G}(\theta)}=\theta$.

Lemma 3. Let $A$ be an abelian normal subgroup of $G$ such that $G / A$ is abelian. Then any nonlinear irreducible character of $G$ is induced from some linear character of some normal subgroup $N$ of $G$ with $A \leq N \leq G$.

Proof. Since $G / A$ is abelian, $G^{\prime} \leq A$.

Let $G=G_{0}>G_{1}>G_{2}>\cdots>A$ be a chief series of $G$ through $A$. Each chief factor $G_{i} / G_{i+1}$ has prime order. Let $\chi$ be any nonlinear irreducible character of $G$. $\chi_{A}$ reduces as $A$ is abelian, so we choose $k$ such that $\chi_{G_{K}}$ is irreducible but $\chi_{G_{k+1}}$ reduces. Clearly $\chi_{G_{K}}$ is invariant in $G$. By Theorem A, $\chi_{G_{k+1}}=\sum_{i=1}^{P} \theta_{i}$ where $P=\left[G_{k}: G_{k+1}\right.$ is a prime, and $\theta_{i}$ are distinct irreducible character of $G_{k+1}$. Thus the inertia group $T_{1}=I_{G}\left(\theta_{1}\right.$ is a proper subgroup of $G$. By Lemma C, $\exists \gamma \in \operatorname{Irr}\left(T_{1}\right.$ such that $\gamma^{G}=\chi$ and $\gamma_{G_{k+1}}=\theta_{1}$.

Note that $T_{1} \triangleleft G$ as $T_{1} \geq A \geq G^{\prime}$, so if $\gamma$ is linear, the proof is complete. If $\gamma$ is nonlinear, then consider the situation where $A$ is an abelian normal subgroup of $T_{1}$, and $T_{1} / A$ is abelian. By induction on $|G|$, every nonlinear irreducible character of $T_{1}$ is induced from some linear character of some normal subgroup $N$ of $T_{1}$ with $A \leq N \leq T_{1}$. In particular, $\exists \theta \in \operatorname{Irr}(N), \theta(1)=1$ and $\theta^{T_{1}}=\gamma$. Thus $\theta^{G}=\gamma^{G}=\chi$. Note that $N \triangleleft G$ as $G^{\prime} \leq N$. This complete the proof.

Corollary 4. Let $G$ be a metabelian group. Then any nonlinear irreducible character of $G$ is induced from a linear character of some normal subgroup $N$ of $G$ with $G^{\prime}<N$.

Proof. Take $A=G^{\prime}$ in Lemma 3.

Theorem 2. Let $G$ be a nonabelian 2-group with property (*). Then

(1) All nonlinear irreducible characters of $G$ have the same degree equals $[G: Z(G)]^{\frac{1}{2}}$.

(2) All maximal abelian normal subgroups of $G$ have the same order. Fix any maximal abelian normal subgroup $A$, then any nonlinear irreducible character of $G$ is induced from some linear character of $A$.

(3) $\Phi(G) \leq Z(G)$, where $\Phi(G)$ is the Frattini subgroup of $G$.

Proof. By Theorem 1, $\left|G^{\prime}\right|=2$, so $G$ is metabelian. Let $\chi$ be any nonlinear irreducible character of $G$. Cor 4 asserts that $\chi$ is induced from some linear character $\theta$ of some normal subgroup $N$ with $G^{\prime} \leq N \leq G$. By Clifford's Theorem, $\chi_{N}=k \sum_{i=1}^{s} \theta_{i}$, where $\theta_{i}$ are conjugate to $\theta_{1}=\theta$. Thus $\operatorname{Ker} \chi \geq N^{\prime}$. As $\left|G^{\prime}\right|=2$, either $N^{\prime}=G^{\prime}$ or 
$N^{\prime}=\left\{e \cdot N^{\prime}=G^{\prime}\right.$ means Ker $\chi \geq G^{\prime}$ contradicting the nonlinearity of $\chi$. So $N^{\prime}=\{e$ and $N$ is abelian. Clearly $N$ is maximal abelian or else $\theta^{G}$ reduces.

If $\chi_{1}, \chi_{2} \in \operatorname{Irr}(G)$ are nonlinear. By the above argument, there exists maximal abelian normal subgroup $N_{1}, N_{2}$ of $G$ such that $\chi_{1}=\theta_{1}^{G}, \chi_{2}=\theta_{2}^{G}$ where $\theta_{i} \in \operatorname{Irr}\left(N_{i}\right.$ $i=1,2$. By Theorem B, $\chi_{1}(1) \mid\left[G: N_{2}\right]=\chi_{2}(1)$ and $\left.\chi_{2}(1) \mid G: N_{1}\right]=\chi_{1}(1)$ proving $\chi_{1}(1)=\chi_{2}(1)$. This proved that any two nonlinear irreducible character of $G$ have the same degree. Using the equality $\sum_{\chi \in \operatorname{Irr}(G)} \chi(1)^{2}=|G|$, we get $\left[G: G^{\prime}\right]+\left(\frac{|G|+|Z(G)|}{2}-\right.$ $\left.\left[G ; G^{\prime}\right]\right) \chi(1)^{2}=|G|$ and this forces $X(1)^{2}=[G: Z(G)$. This proved (1). To prove (2), let $A$ be any maximal abelian normal subgroup of $G$ and $\chi$ any nonlinear irreducible character of $G$. By Lemma $3, \exists N \triangleleft G$ such that $A \leq N \leq G$ and $\chi=\theta^{G}$ with $\theta$ a linear character of $N$. Note that $G^{\prime} \leq A \leq N$. By the same argument as in the first part of the proof, we see that $N$ is abelian and so $N=A$ as $A$ is maximal abelian normal in $G$. This in particular shows that $[G: A]=\chi(1)$, or $|A|=|G| / \chi(1)$. This proves (2).

$\forall x \in G$, if $x^{2} \notin Z(G)$, then $\exists y \in G$ such that $x^{2} y x^{-2} y^{-1} \neq e$. So $x y x^{-1} y^{-1} \neq e$. As $\left|G^{\prime}\right|=2$, we must have $x^{2} y x^{-2} y^{-1}=x y x^{-1} y^{-1}$ and this forces $x y=y x$, a contradiction. Thus $G^{2} \leq Z(G)$ and $\Phi(G)=G^{\prime} G^{2} \subseteq Z(G)$. Theorem 2 shows strong similarity between nonabelian 2-group with property $(*)$ and
extra special 2-group.

A group $G$ is a central product of its subgroups $N_{1}, \ldots, N_{k}$ if $G=N_{1} N_{2} \ldots N_{k}$ and for each $x \in N_{i}, y \in N_{j}, i \neq j$, then $x^{-1} y^{-1} x y=e$. See [1] $\operatorname{pg} 179$.

Theorem 3. If $G$ is a nonabelian 2-group satisfying property (*), then $G$ is a central product of 2-generator groups and an abelian group.

Proof. Without loss of generality, we assume that $G$ is directly indecomposable. Let $x \in G \backslash Z(G)$, so $\left[G: C_{G}(x)\right]=2$. Choose $y \in G \backslash C_{G}(x)$, and let $A=\langle x, y\rangle$. Obviously, $\left[G: C_{G}(y)\right]=2$ and hence $C_{G}(A)$ has index 4 in $G$. As $[A: Z(A)] \geq 4$, we have

$$
\left|A C_{G}(A)\right|=\frac{|A|\left|C_{G}(A)\right|}{|Z(A)|} \geq|G|
$$

So $A C_{G}(A)=G$. We conclude that $G$ is a central product of $A$ and $C_{G}(A)$. Now $C_{G}(A)$ being a subgroup of $G$ also satisfies property $(*)$. If it is abelian, the proof is
complete. Otherwise, use induction on $|G|$ to con complete. Otherwise, use induction on $|G|$ to conclude that $C_{G}(A)$ is a central product of 2-generator gropus with an abelian group. $C_{G}(A)=A_{2} A_{3} \cdots A_{s} L$ where $A_{i}$ are 2-generator groups and $L$ is abelian. So $G=A A_{2} A_{3} \cdots A_{s} L$ is a central product of 2-generator groups and an abelian group as required. This complete the proof.

\section{References}

[1] L. Dornhoff, Group Representation Theory, Part A. Marcel Dekker, New York, 1971.

[2] G. A. How, Special Classes of Monomial Groups III, Chinese Journal of Mathematics 12
(1984), 199-211.

[3] I. M. Isaacs, Character Theory of Finite Groups, Academic Press, New York, San Franscisco,
London, 1976. 
[4] I. M. Isaacs and D. Passman, Groups with Representations of bounded degree, Canad. J. Math. 16 (1964), 299-309.

School of Mathematical Sciences, University Sains Malaysia, 11800 Pulau Pinang, Malaysia. Department of Applied Mathematics, Feng Chia University, Taichung, Taiwan. 